\title{
LINEAR POISSON STRUCTURES AND HOM-LIE ALGEBROIDS
}

\author{
ESMAEIL PEYGHAN, AMIR BAGHBAN, AND ESA SHARAHI
}

\begin{abstract}
Considering Hom-Lie algebroids in some special cases, we obtain some results of Lie algebroids for Hom-Lie algebroids. In particular, we introduce the local splitting theorem for Hom-Lie algebroids. Moreover, linear Hom-Poisson structure on the dual Hom-bundle will be introduced and a one-to-one correspondence between Hom-Poisson structures and Hom-Lie algebroids will be presented. Also, we introduce Hamiltonian vector fields by using linear Poisson structures and show that there exists a relation between these vector fields and the anchor map of a Hom-Lie algebroid.
\end{abstract}

\section{INTRODUCTION}

Hom-Lie algebroids were introduced by Laurent-Gengoux and Teles in [4] using the notion of Hom-Gerstenhaber algebras. Afterwards, in [1], Cai, Liu, and Sheng modified the definition of a Hom-Lie algebroid and gave its equivalent dual description. A Hom-Lie algebroid has its own geometric meaning and interesting examples, and it is more than a formal generalization of a Lie algebroid. Recently, many researchers have been interested in studying the algebraic and geometric concepts on Lie algebroids and Hom-Lie algebroids ([3, 5, 7, 8, 10, 11, 12, 13, 14, 15, 16]).

In [1] the authors could fix the definition of Hom-Lie algebroid in a more suitable way by introducing the notion of Hom-bundle. In this sense, there is a fundamental example. Let $\varphi: M \rightarrow M$ be any diffeomorphism. Then the pull back bundle $\varphi^{!} \mathrm{T} M$ of the tangent Lie algebroid TM is a Hom-Lie algebroid. This example is based on the concept of the Hom bundle, and using it the authors introduced the Hom-Poisson tensor structures. Therefore, we see the naturality of this definition of Hom-Lie algebroids. In this paper, we consider the definition of Hom-Lie algebroid given in [1].

Many results in Hamiltonian dynamics are indebted to Poisson geometry, where they serve as phase spaces. Though Poisson geometry was an outcome of symplectic geometry, it is a powerful theory in mathematics now. Lots of relations with other fields - like Hamiltonian dynamics, representation theory, quantum physics, dynamical and integrable systems - make Poisson geometry a simple to use and essential approach. Indeed, translating the concepts to Poisson literature, may

2010 Mathematics Subject Classification. 17B99, 53D17.

Key words and phrases. Hamiltonian vector field; Hom-Lie algebroid; linear Hom-Poisson structure. 
reduce the amount of computations or may solve the problem in a more technical way. All of this paper's discussions can be reduced to the Lie algebroid case. Lie algebroids are just some fiber-wise linear Poisson structures. This kind of approach is different by considering them as infinitesimal versions of Lie groupoids. Here, one can see that Poisson makes a correlation between commutative and noncommutative objects [2].

The structure of this paper is organized as follows: In Section 2, we recall the notions of Hom-algebra and Hom-Lie algebroid and present some examples of these concepts. In Section 3, we obtain some results on Hom-Lie algebroids in special cases. In particular, we present the locally splitting theorem for HomLie algebroids. Section 4 is devoted to the study of linear Poisson structures and Hamiltonian vector fields on Hom-Lie algebroids.

\section{Preliminaries}

In this section, we present some notions and results about Hom-Lie algebras and Hom-Lie algebroids (see [1, 9, 10] for more details).

A triple $\left(\mathfrak{g},[\cdot, \cdot], \phi_{\mathfrak{g}}\right)$ consisting of a linear space $\mathfrak{g}$, a bilinear map (bracket) $[\cdot, \cdot]: \mathfrak{g} \times \mathfrak{g} \rightarrow \mathfrak{g}$, and an algebra morphism $\phi_{\mathfrak{g}}: \mathfrak{g} \rightarrow \mathfrak{g}$ satisfying

$$
[u, v]=-[v, u] \quad \text { and } \circlearrowleft_{u, v, w}\left[\phi_{\mathfrak{g}}(u),[v, w]\right]=0, \quad u, v, w \in \mathfrak{g},
$$

is called a Hom-Lie algebra (the second equation is called Hom-Jacobi identity). The Hom-Lie algebra $\left(\mathfrak{g},[\cdot, \cdot], \phi_{\mathfrak{g}}\right)$ is called regular (involutive), if $\phi_{\mathfrak{g}}$ is non-degenerate (satisfies $\left.\phi_{\mathfrak{g}}{ }^{2}=1\right)$. A representation of $\left(\mathfrak{g},[\cdot, \cdot], \phi_{\mathfrak{g}}\right)$ is a triple $(V, A, \rho)$ where $V$ is a vector space, $A \in g l(V)$ and $\rho: \mathfrak{g} \rightarrow g l(V)$ is a linear map satisfying

$$
\left\{\begin{array}{l}
\rho\left(\phi_{\mathfrak{g}}(u)\right) \circ A=A \circ \rho(u), \\
\rho\left([u, v]_{\mathfrak{g}}\right) \circ A=\rho\left(\phi_{\mathfrak{g}}(u)\right) \circ \rho(v)-\rho\left(\phi_{\mathfrak{g}}(v)\right) \circ \rho(u),
\end{array}\right.
$$

for any $u, v \in \mathfrak{g}$.

Let $A$ be a vector bundle of rank $r$ over the manifold $M$. A Hom-bundle is a triple $\left(A \rightarrow M, \varphi, \phi_{A}\right)$ consisting of a vector bundle $A \rightarrow M$, a smooth map $\varphi: M \rightarrow M$, and an algebra morphism $\phi_{A}: \Gamma(A) \rightarrow \Gamma(A)$ satisfying

$$
\phi_{A}(f X)=\varphi^{*}(f) \phi_{A}(X),
$$

for any $X \in \Gamma(A)$ and $f \in C^{\infty}(M)$. If $\varphi$ is a diffeomorphism and $\phi_{A}$ is an invertible map, then the Hom-bundle $\left(A \rightarrow M, \varphi, \phi_{A}\right)$ is called invertible. An example of a Hom-bundle is $\left(\Gamma\left(\varphi^{!} \mathrm{T} M\right), \varphi, A d_{\varphi^{*}}\right)$, where $A d_{\varphi^{*}}: \Gamma\left(\varphi^{!} \mathrm{T} M\right) \rightarrow \Gamma\left(\varphi^{!} \mathrm{T} M\right)$ is given by

$$
A d_{\varphi^{*}}(X)=\varphi^{*} \circ X \circ\left(\varphi^{*}\right)^{-1}, \quad \forall X \in \Gamma\left(\varphi^{!} \mathrm{T} M\right) .
$$

Remark 2.1. If $\phi_{A}: \Gamma(A) \rightarrow \Gamma(A)$ satisfies 2.1), then we have the bundle morphism $\phi_{A}: \varphi^{!} A \rightarrow A$ given by $\phi_{A}\left(m, X_{\varphi(m)}\right)=\left(m, \phi_{A}(X)_{m}\right)$, for all $m \in M$. The converse of this assertion is also true. 
Definition 2.2. Let $\left(A \rightarrow M, \varphi, \phi_{A}\right)$ and $\left(B \rightarrow M, \varphi, \phi_{B}\right)$ be two Hom-bundles. A bundle map $\rho: A \rightarrow B$ is called a morphism of Hom-bundles if the following condition holds:

$$
\rho \circ \phi_{A}=\phi_{B} \circ \rho .
$$

The linear map $\phi_{A}: \Gamma(A) \rightarrow \Gamma(A)$ given in a Hom-bundle $\left(A \rightarrow M, \varphi, \phi_{A}\right)$ can be extended to a linear map from $\Gamma\left(\wedge^{k} A\right)$ to $\Gamma\left(\wedge^{k} A\right)$ for which we use the same notation $\phi_{A}$ via

$$
\phi_{A}(X)=\phi_{A}\left(X_{1}\right) \wedge \cdots \wedge \phi_{A}\left(X_{k}\right), \quad \forall X=X_{1} \wedge \cdots \wedge X_{k} \in \Gamma\left(\wedge^{k} A\right) .
$$

If the Hom-bundle $A$ is invertible, then the inverses of $\varphi$ and $\phi_{A}$ are denoted by $\varphi^{-1}$ and $\phi_{A}^{-1}$, respectively. Also it is easy to see that

$$
\phi_{A}^{-1}(f X)=\left(\varphi^{*}\right)^{-1}(f) \phi_{A}^{-1}(X), \quad \forall f \in C^{\infty}(M), X \in \Gamma\left(\wedge^{k} A\right) .
$$

So $\left(A \rightarrow M, \varphi^{-1}, \phi_{A}^{-1}\right)$ is a Hom-bundle. We consider $\phi_{A}^{\dagger}: \Gamma\left(\wedge^{k} A^{*}\right) \rightarrow \Gamma\left(\wedge^{k} A^{*}\right)$ defined by

$$
\left(\phi_{A}^{\dagger}(\xi)\right)(X)=\varphi^{*} \xi\left(\phi_{A}^{-1}(X)\right), \quad \forall X \in \Gamma\left(\wedge^{k} A\right), \xi \in \Gamma\left(\wedge^{k} A^{*}\right) .
$$

From the above equation, we can conclude that

$$
\phi_{A}^{\dagger}(f \xi)=\varphi^{*}(f) \phi_{A}^{\dagger}(\xi) .
$$

Therefore $\left(\wedge^{k} A^{*}, \varphi, \phi_{A}^{\dagger}\right)$ is a Hom-bundle.

Remark 2.3. When $k=1$, similar to Remark 2.1 we have the correspondence bundle map $\phi_{A}^{\dagger}\left(m, \omega_{\varphi(m)}\right)=\left(m, \phi_{A}^{\dagger}(\omega)_{m}\right)$, for all $m \in M$, for $\phi_{A}^{\dagger}: \Gamma\left(A^{*}\right) \rightarrow \Gamma\left(A^{*}\right)$ satisfying 2.2.

Definition $2.4([1])$. A Hom-Lie algebroid is a multiple $\left(A, \varphi, \phi_{A},[\cdot, \cdot]_{A}, a_{A}\right)$ such that $\left(A \rightarrow M, \varphi, \phi_{A}\right)$ is a Hom-bundle, $\left(\Gamma(A),[\cdot, \cdot]_{A}, \phi_{A}\right)$ is a Hom-Lie algebra on the section space $\Gamma(A), a_{A}: A \rightarrow \varphi^{!} \mathrm{T} M$ is a bundle map called the anchor map and moreover, we have

$[X, f Y]_{A}=\varphi^{*}(f)[X, Y]_{A}+a_{A}\left(\phi_{A}(X)\right)(f) \phi_{A}(Y), \quad \forall X, Y \in \Gamma(A), \forall f \in C^{\infty}(M)$, when $a_{A}: \Gamma(A) \rightarrow \Gamma\left(\varphi^{!} \mathrm{T} M\right)$ is the representation of the Hom-Lie algebra $(\Gamma(A)$, $\left.[\cdot, \cdot]_{A}, \phi_{A}\right)$ on $C^{\infty}(M)$ with respect to $\varphi^{*}$ induced by the anchor map.

It is easy to see that $\left(\varphi^{!} \mathrm{T} M, \varphi, A d_{\varphi^{*}},[\cdot, \cdot]_{\varphi^{*}}, i d\right)$ is a Hom-Lie algebroid [1], where $[X, Y]_{\varphi^{*}}=\varphi^{*} \circ X \circ\left(\varphi^{*}\right)^{-1} \circ Y \circ\left(\varphi^{*}\right)^{-1}-\varphi^{*} \circ Y \circ\left(\varphi^{*}\right)^{-1} \circ X \circ\left(\varphi^{*}\right)^{-1}, \quad \forall X, Y \in \varphi^{!} \mathrm{T} M$. Let $\left(A, \varphi, \phi_{A},[\cdot, \cdot]_{A}, a_{A}\right)$ and $\left(B, \varphi, \phi_{B},[\cdot, \cdot]_{B}, a_{B}\right)$ be two Hom-Lie algebroids over $M$. A base-preserving morphism with the same base from $A$ to $B$ is a bundle map $\rho: A \rightarrow B$ such that

$$
a_{B} \circ \rho=a_{A}, \quad \rho \circ \phi_{A}=\phi_{B} \circ \rho, \quad \rho\left([X, Y]_{A}\right)=[\rho(X), \rho(Y)]_{B} .
$$

If $\varphi$ is a diffeomorphism, it is easy to see that the map $a_{A}$ is a morphism between Hom-Lie algebroids, i.e.,

$$
a_{A}\left(\phi_{A}(X)\right)=A d_{\varphi^{*}}\left(a_{A}(X)\right), \quad a_{A}\left([X, Y]_{A}\right)=\left[a_{A}(X), a_{A}(Y)\right]_{\varphi^{*}} .
$$




\section{LOCAL SPLitTing THEOREM}

Let $x^{i}: U \subseteq M \longrightarrow \mathbb{R}$ be a coordinate system on $M, \Psi_{\alpha}: U \subseteq M \longrightarrow A$ and $\Psi^{\alpha}: U \subseteq M \longrightarrow A^{*}$ be a local basis of sections of $A$ and $A^{*}$, respectively. Let $\left(\left(x^{1}, \ldots, x^{m}, \xi_{1}, \ldots, \xi_{r}\right), \pi^{-1}(U)\right)$ be the coordinate system on $A^{*}$, where $\xi_{\alpha}: A^{*} \rightarrow$ $\mathbb{R}$ is defined by $\xi_{\beta}\left(\xi_{\alpha} \Psi^{* \alpha}\right)=\xi_{\beta}$. So, the local vector fields on $A^{*}$ associated to this coordinate system are denoted by $\frac{\partial}{\partial x^{i}}, \frac{\partial}{\partial \xi_{\alpha}}$.

Suppose that $\phi_{A}$ is diagonalizable on $A$, that is, there exists a basis of sections such that its matrix is diagonal with respect to it. Let $f_{1}, \ldots, f_{k}$ be locally smooth functions on an open subset $U \subset M$ which are the eigenvalues of $\phi_{A}$ at every point. We denote the eigenspace of $\phi_{A}$ related to $f_{i}$ at $p$ by $A_{i p}$. Suppose $A_{i}=\cup_{p} A_{i p}$. Since $\phi_{A}: A \rightarrow A$ is a smooth function between two smooth manifolds, $A_{i}$ has constant rank for all $i=1, \ldots, k$. Therefore, they are distributions of $A$. Now we can state the following theorem in order to classify $\phi_{A}$.

Theorem 3.1. Let $\left(A, \operatorname{Id}_{M}, \phi_{A}=f \operatorname{Id}_{A},[\cdot, \cdot]_{A}, a_{A}\right)$ be a Hom-Lie algebroid where $f: M \rightarrow M$ is a smooth function. If the rank of $a_{A}$ is greater than 0 then $f$ must be constant 1 on $M$. Moreover, when $\varphi=\operatorname{Id}_{M}$ and $\phi_{A}$ is arbitrary, if the rank of $a_{A}$ is greater than zero on every $A_{i}$ then $\phi_{A}$ is the identity isomorphism.

Proof. Using the equation $a_{A}\left(\phi_{A}(X)\right) \circ \varphi^{*}=\varphi^{*} \circ a_{A}(X)$ and considering $\varphi=\operatorname{Id}_{M}$ and $\phi_{A}=f \operatorname{Id}_{A}$ one can get $f a_{A}(X)=a_{A}(X)$. Since $a_{A}$ is a non-zero bundle morphism the result of the first part can be achieved, i.e., $f \equiv 1$. For the second part, let $X_{i} \in \Gamma\left(A_{i}\right)$ such that $a_{A}\left(X_{i}\right) \neq 0$. If $\phi_{A}\left(X_{i}\right)=f_{i} X_{i}$ then using the equation $a_{A}\left(\phi_{A}(X)\right) \circ \varphi^{*}=\varphi^{*} \circ a_{A}(X)$ we get $f_{i} a_{A}\left(X_{i}\right)=a_{A}\left(X_{i}\right)$. This shows that $f_{i} \equiv 1$ and so $\phi_{A} \equiv \operatorname{Id}_{A}$.

Definition 3.2. An $a_{A}$-section $S$ of $\varphi^{!} \mathrm{T} M$ is a section of $\varphi^{!} \mathrm{T} M$ such that there exists a section $\Psi$ of $A$ satisfying $a_{A}(\Psi)=S$. We denote the set of such sections by $\Gamma_{A}\left(\varphi^{!} \mathrm{T} M\right)$. It is easy to see that $\Gamma_{A}\left(\varphi^{!} \mathrm{T} M\right)$ is a $C^{\infty}(M)$-module.

Theorem 3.3. If $\phi_{A}=f \operatorname{Id}_{A}$, then $\varphi_{*}: \Gamma_{A}\left(\varphi^{!} \mathrm{T} M\right) \rightarrow \Gamma_{A}\left(\varphi^{!} \mathrm{T} M\right)$ is a multiple of the identity.

Proof. Let $X$ be a section of $A$. Using the relation $a_{A}\left(\phi_{A}(X)\right) \circ \varphi^{*}=\varphi^{*} \circ a_{A}(X)$ we get

$$
f(p)\left(a_{A}(X)\right)_{p}(g \circ \varphi)=a_{A}(X)_{\varphi(p)}(g),
$$

for $g \in C^{\infty}(M)$ and $p \in M$. So, one can get

$$
d_{\varphi(p)} g\left(f(p) \varphi_{* p}\left(a_{A}\left(X_{p}\right)\right)-a_{A}(X)_{\varphi(p)}\right) .
$$

Since $g$ was an arbitrary smooth function on $M$ we derive that

$$
f(p) \varphi_{* p}\left(a_{A}\left(X_{p}\right)\right)-a_{A}(X)_{\varphi(p)}=0 .
$$

Since $\phi_{A}$ is an isomorphism, $f(p) \neq 0$ and then we have

$$
\varphi_{* p}\left(a_{A}\left(X_{p}\right)\right)=\frac{1}{f(p)} a_{A}(X)_{\varphi(p)},
$$

and the proof is completed. 
Proposition 3.4. If $\varphi$ is the identity mapping on $M$ then we have

$$
a_{A}[X, Y]_{A}=\left[a_{A}(X), a_{A}(Y)\right]_{M} .
$$

Proof. If $\varphi$ is the identity then

$$
a_{A}\left(\phi_{A}(X)\right)=a_{A}(X),
$$

and

$$
a_{A}[X, Y]_{A}=a_{A}\left(\phi_{A}(X)\right) a_{A}(Y)-a_{A}\left(\phi_{A}(Y)\right) a_{A}(X) .
$$

If we set $a_{A}(Y)=a_{A}(\phi(Y))$ and $a_{A}(X)=a_{A}(\phi(X))$ we get

$$
a_{A}[X, Y]_{A}=a_{A}(X) a_{A}(Y)-a_{A}(Y) a_{A}(X),
$$

but this is equivalent to

$$
a_{A}[X, Y]_{A}=\left[a_{A}(X), a_{A}(Y)\right]_{M} .
$$

Theorem 3.5. Let $\left(A, \varphi, \phi_{A},[\cdot, \cdot]_{A}, a_{A}\right)$ be a Hom-Lie algebroid and $x_{0}$ be a point in $M$ such that the rank of $\left.a_{A}\right|_{A_{t}}$ at $x_{0}$ is $r_{t}$, the dimension of fibers of $A_{t}$ is $\alpha_{t}$, and $A_{t}$ is the eigenspace of $\phi_{A}$ with respect to the eigenvalue $f_{t}$. Then there exist coordinates $\left(x^{i}, y^{j}\right)$, where $i=1, \ldots, r_{t}$ and $j=r_{t}+1, \ldots, m$ on a neighborhood $U$ of $x_{0}$ and a basis of sections $\left\{\Psi_{1}, \ldots, \Psi_{\alpha_{t}}\right\}$ of $A_{t}$ over $U$ satisfying

$$
\begin{gathered}
\left.a_{A}\right|_{A_{t}}\left(\Psi_{i}\right)=\frac{\partial}{\partial x^{i}}, \quad i=1, \ldots, r_{t}, \\
\left.a_{A}\right|_{A_{t}}\left(\Psi_{\alpha}\right)=\sum_{j} a_{\alpha}^{j} \frac{\partial}{\partial y^{j}}, \quad \alpha=r_{t}+1, \ldots, \alpha_{t},
\end{gathered}
$$

where $a_{\alpha}^{j} \in C^{\infty}(U)$ are smooth functions depending only on the $y$ 's and vanishing at $x_{0}$; namely $a_{\alpha}^{j}=a_{\alpha}^{j}\left(y^{k}\right)$ and $a_{\alpha}^{j}(0)=0$. Moreover,

$$
\left[\Psi_{\alpha}, \Psi_{\beta}\right]=\sum_{\gamma} c_{\alpha \beta}^{\gamma} \Psi_{\gamma}
$$

where $c_{\alpha \beta}^{\gamma} \in C^{\infty}(U)$ vanish if $\gamma \leq r_{t}$ and satisfy

$$
\begin{aligned}
\sum_{\gamma>r_{t}} a_{\gamma}^{j} \frac{\partial c_{\alpha \beta}^{\gamma}}{\partial x^{i}} & =0, \\
\sum_{\gamma>r_{t}} c_{\alpha \beta}^{\gamma} a_{\gamma}^{j} \frac{\partial f_{t}}{\partial y^{j}} & =0,
\end{aligned}
$$

where $f_{t}$ is the eigenvalue of $\phi_{A}$ related to the eigenspace $A_{t}$.

Proof. Our proof is based on the rank of $\left.a_{A}\right|_{A_{t}}$. By assuming the rank of $\left.a_{A}\right|_{A_{t}}$ to be $r_{t}$ we give a proof by induction on $r_{t}$ (indeed, we don't change any construction here but we can suppose that there exists some subspace of $A_{t}$ so that the restriction of $\left.a_{A}\right|_{A_{t}}$ to this subspace is of rank $k<r_{t}$ ). Suppose we have all results of the theorem for $0 \leq k<r_{t}$ and assume we have constructed coordinates $\left(x^{i}, \tilde{y}^{j}\right)$ where 
$i \leq k$ and $k<j \leq m$ on $U \subset M$ and a basis of sections $\left\{\Psi_{i}, \tilde{\Psi}_{\alpha}\right\}$ for $A_{t}$ over $U$, where $i \leq k$ and $k<\alpha \leq \alpha_{t}\left(\alpha_{t}\right.$ is the rank of $\left.A_{t}\right)$ such that

$$
a_{A}\left(\Psi_{i}\right)=\frac{\partial}{\partial x^{i}}, \quad a_{A}\left(\tilde{\Psi}_{\alpha}\right)=a_{\alpha}^{j} \frac{\partial}{\partial \tilde{y}^{j}},
$$

where $a_{\alpha}^{j}$ depend only on the $\tilde{y}$ 's. Since $r_{t}>k$, there exists an $\alpha$ such that the section $a_{A}\left(\tilde{\Psi}_{\alpha}\right)$ does not vanish at $x_{0}$ (since the rank of $\left.a_{A}\right|_{A_{t}}$ is supposed to be $r_{t}$, which is greater than $k$ ). We can assume that $\alpha=k+1$ and we set $\Psi_{k+1}=\tilde{\Psi}_{k+1}$. We can perform a change of coordinates

$$
x^{k+1}=x^{k+1}\left(\tilde{y}^{k+1}, \ldots, \tilde{y}^{m}\right), \quad y^{j}=y^{j}\left(\tilde{y}^{k+1}, \ldots, \tilde{y}^{m}\right),
$$

such that

$$
\begin{gathered}
a_{A}\left(\Psi_{k+1}\right)=\frac{\partial}{\partial x^{k+1}} \\
a_{A}\left(\tilde{\Psi}_{\alpha}\right)=a_{\alpha}^{k+1} \frac{\partial}{\partial x^{k+1}}+a_{\alpha}^{k+2} \frac{\partial}{\partial y^{k+2}}+\cdots .
\end{gathered}
$$

Note that such change of coordinates can happen as $a_{A}\left(\Psi_{k+1}\right)\left(x_{0}\right) \neq 0$. If we change $\tilde{\Psi}_{\alpha}$ with $\tilde{\Psi}_{\alpha}-a_{\alpha}^{k+1} \Psi_{k+1}$ we get

$$
a_{A}\left(\tilde{\Psi}_{\alpha}\right)=a_{\alpha}^{l} \frac{\partial}{\partial y^{l}}
$$

where $a_{\alpha}^{l}=a_{\alpha}^{l}\left(x^{k+1}, y^{k+2}, \ldots, y^{m}\right)$ and $l=k+2, \ldots, m$. Since $r_{t} \geq 1$, according to Theorem 3.1 we have $f_{t} \equiv 1$. So, for all sections $X, Y$ on $A_{t}$ we have

$$
a_{A}[X, Y]_{A}=\left[a_{A}(X), a_{A}(Y)\right]_{M},
$$

and this shows that

$$
\left[\Psi_{k+1}, \tilde{\Psi}_{\alpha}\right]_{A}=\sum_{\gamma>k+1} c_{k+1, \alpha}^{\gamma} \tilde{\Psi}_{\gamma} .
$$

By setting $a_{A}$ in two sides of (3.5) and using (3.4) we get

$$
\frac{\partial a_{\alpha}^{l}}{\partial x^{k+1}} \frac{\partial}{\partial y^{l}}=\sum_{\gamma>k+1} c_{k+1, \alpha}^{\gamma} a_{\gamma}^{l} \frac{\partial}{\partial y^{l}},
$$

which yields

$$
\frac{\partial a_{\alpha}^{l}}{\partial x^{k+1}}=\sum_{\gamma>k+1} c_{k+1, \alpha}^{\gamma} a_{\gamma}^{l} .
$$

Similar to Fernandes' argument to solve this ODE we get a coordinate system $\left(x^{i}, y^{j}\right)$ and sections $\{\Psi\}_{i=1}^{\alpha_{t}}$ satisfying the relations 3.1 and 3.2. Using the equation

$$
a_{A}[X, Y]_{A}=\left[a_{A}(X), a_{A}(Y)\right]_{M}
$$


one can easily check that $c_{\alpha \beta}^{\gamma} \in C^{\infty}(U)$ vanish if $\gamma \leq r_{t}$ in (3.3). Using the Hom-Jacobi identity, one can get the following for $i \leq r_{t}$ and $r_{t} \leq \alpha, \beta \leq \alpha_{t}$ :

$$
\begin{aligned}
a_{A}\left[f_{t} \Psi_{i},\left[\Psi_{\alpha}, \Psi_{\beta}\right]_{A}\right]_{A}= & f_{t}\left[f_{t}\left[\frac{\partial}{\partial x^{i}}, a_{\alpha}^{l} \frac{\partial}{\partial y^{l}}\right]_{A}, f_{t} a_{A} \Psi_{\beta}\right]_{A} \\
& +f_{t}\left[f_{t} a_{A} \Psi_{\alpha}, f_{t}\left[\frac{\partial}{\partial x^{i}}, a_{\beta}^{l} \frac{\partial}{\partial y^{l}}\right]_{A}\right]_{A}=0 .
\end{aligned}
$$

On the other hand,

$$
\begin{aligned}
& a_{A}\left[f_{t} \Psi_{i},\left[\Psi_{\alpha}, \Psi_{\beta}\right]_{A}\right]_{A}=a_{A}\left[f_{t} \Psi_{i}, \sum_{\gamma>r_{t}} c_{\alpha \beta}^{\gamma} \Psi_{\gamma}\right]_{A}, \\
& f_{t}\left[f_{t} \frac{\partial}{\partial x^{i}}, \sum_{\gamma>r_{t}} c_{\alpha \beta}^{\gamma} a_{\gamma}^{j} \frac{\partial}{\partial y^{j}}\right]_{A}=\left(f_{t}\right)^{2} \sum_{\gamma>r_{t}} a_{\gamma}^{j} \frac{\partial c_{\alpha \beta}^{\gamma}}{\partial x^{i}} \frac{\partial}{\partial y^{j}}+f_{t} \sum_{\gamma>r_{t}} c_{\alpha \beta}^{\gamma} a_{\gamma}^{j} \frac{\partial f_{t}}{\partial y^{j}} \frac{\partial}{\partial x^{i}}=0 .
\end{aligned}
$$

So, we have

$$
\sum_{\gamma>r_{t}} a_{\gamma}^{j} \frac{\partial c_{\alpha \beta}^{\gamma}}{\partial x^{i}}=0, \quad \sum_{\gamma>r_{t}} c_{\alpha \beta}^{\gamma} a_{\gamma}^{j} \frac{\partial f_{t}}{\partial y^{j}}=0 .
$$

Note that, in general, $r_{t}$ can be zero and so it is not necessary that we have $f_{t} \equiv 1$.

Remark 3.6. Using the above theorem and the equation

$$
\rho[X, Y]_{\mathfrak{g}}=\rho\left(\phi_{\mathfrak{g}}(X)\right) \circ \rho(Y)-\rho\left(\phi_{\mathfrak{g}}(Y)\right) \circ \rho(X),
$$

one can deduce a Fernandes-like result. Indeed, if $\left\{\Psi_{1}^{i}, \ldots, \Psi_{\alpha_{i}}^{i}\right\}$ is a basis of sections for $A_{i}$ and $\left(x_{i}^{1}, \ldots, x_{i}^{r_{i}}, y_{i}^{r_{i}+1}, \ldots, y_{i}^{m}\right)$ is a coordinate system about $x_{0} \in M$ for each $A_{i}$ satisfying the last theorem and if the rank of $a_{A}$ is $q$ at $x_{0}$ then there exist coordinates $\left(x^{i}, y^{j}\right)$ and a basis of sections $\left\{\Psi^{\alpha}\right\}_{1}^{r}$ of $A$ over $U$ satisfying

$$
\begin{aligned}
& a_{A}\left(\Psi_{i}\right)=\frac{\partial}{\partial x^{i}}, \quad i=1, \ldots, r, \\
& a_{A}\left(\Psi_{\alpha}\right)=\sum_{j} a_{\alpha}^{j} \frac{\partial}{\partial y^{j}}, \quad \alpha>r,
\end{aligned}
$$

where $r_{i}$ is the rank of $\left.a_{A}\right|_{A_{i}}$ and $\alpha_{i}$ is the rank of $A_{i}$. Indeed, we choose a linearly independent subset of

$$
\left\{\frac{\partial}{\partial x_{1}^{1}}, \frac{\partial}{\partial x_{1}^{2}}, \ldots, \frac{\partial}{\partial x_{1}^{r_{1}}}, \frac{\partial}{\partial x_{2}^{1}}, \ldots, \frac{\partial}{\partial x_{2}^{r_{2}}}, \ldots, \frac{\partial}{\partial x_{k}^{1}}, \ldots, \frac{\partial}{\partial x_{k}^{r_{k}}}\right\},
$$

where $k$ is the number of eigenspaces of $a_{A}$ at $x_{0}$.

Let $A \stackrel{\pi}{\longrightarrow} M$ and $B \stackrel{\nu}{\longrightarrow} M$ be vector bundles and let $T: A \rightarrow B$ be a bundle map which satisfies

$$
\nu \circ T=\pi \text {. }
$$

Now, let $K_{x}=\frac{A_{x}}{\operatorname{ker}_{x} T}$ and let $K=\cup_{x} K_{x}$ for every $x \in M$, where $A_{x}$ is the fiber at $x$ and $\operatorname{ker}_{x} T$ is the kernel of $T$. It is easy to see that if the rank of $T$ on every $A_{x}$ is the same number then $K$ is a subbundle of $A$. Now, we suppose that $A$ is 
a Hom-Lie algebroid and let $B=T M$. We can suppose that $\left.a_{A}\right|_{A_{x}}$ is a surjective linear map. The result is that $K$ is a subbundle of $A$.

Now, let $\rho: K \rightarrow T M$ and $\Delta: K \rightarrow K$ be bundle maps defined by

$$
\rho\left(X_{x}+K_{x}\right)=a_{A}\left(X_{x}\right)
$$

and

$$
\Delta\left(X_{x}+K_{x}\right)=\phi_{A}\left(X_{x}\right)+K_{x} .
$$

Then using $\rho(\Delta X)=\rho\left(\phi_{A}(X)+K\right)=a_{A}\left(\phi_{A}(X)\right)=a_{A}(X)=\rho(X+K)$ we get

$$
\rho(\Delta X)=\rho(X) .
$$

Proposition 3.7. Using the above notations one can prove that $\Delta$ is an identity bundle map.

Proof. It is easy to see that $\rho \circ \Delta=\rho$. If $\Delta\left(X_{x}+K_{x}\right)=\lambda\left(X_{x}+K_{x}\right)$ then using that relation we get $\rho\left(X_{x}+K_{x}\right)=\lambda \rho\left(X_{x}+K_{x}\right)$. Since $\rho$ is one-to-one, we have $\lambda=1$.

Lemma 3.8. $\rho$ and $\Delta$ are well-defined and one-to-one mappings.

Proof. Let $X_{x}-Y_{x}=Z_{x} \in K_{x}$. It is easy to see that $a_{A}\left(X_{x}-Y_{x}\right)=0$ and so $a_{A}\left(X_{x}\right)=\rho\left(X_{x}+K_{x}\right)=\rho\left(Y_{x}+K_{x}\right)=a_{A}\left(Y_{x}\right)$. Now, we show that $\Delta\left(X_{x}+K_{x}\right)=$ $\Delta\left(Y_{x}+K_{x}\right)$, i.e., $\phi_{A}\left(X_{x}-Y_{x}\right) \in K_{x}$. Since, $a_{A} o \phi_{A}=a_{A}$, so $a_{A}\left(\phi_{A}\left(X_{x}-Y_{x}\right)\right)=$ $a_{A}\left(X_{x}-Y_{x}\right)=0$.

Now, let $\rho\left(X_{x}+K_{x}\right)=a_{A}\left(X_{x}\right)=0$. This shows that $X_{x} \in K_{x}$ and the result is that $X_{x}+K_{x}=K_{x}$ and $a_{A}$ is one-to-one. The equation $a_{A} \circ \phi_{A}=a_{A}$ shows that if $\Delta\left(X_{x}+K_{x}\right)=K_{x}$ then $X_{x} \in K_{x}$ and so $\Delta$ is one-to-one.

Theorem 3.9. $\left(K, \rho,[\cdot, \cdot]_{K}, M\right)$ is a Lie algebroid, where $[\cdot, \cdot]_{K}: K \times K \rightarrow K$ is defined by

$$
[X+K, Y+K]_{K}=\rho^{-1}\left[a_{A}(X), a_{A}(Y)\right]_{M} .
$$

Proof. It is obvious that $[\cdot, \cdot]_{K}$ is skew-symmetric. We show that it satisfies the Jacobi identity. Let

$$
\begin{aligned}
L=\left[X+K,[Y+K, Z+K]_{K}\right]_{K} & +\left[Z+K,[X+K, Y+K]_{K}\right]_{K} \\
+ & {\left[Y+K,[Z+K, X+K]_{K}\right]_{K} . }
\end{aligned}
$$

Then we have

$$
\begin{aligned}
\rho L=\left[a_{A}(X),\left[a_{A}(Y), a_{A}(Z)\right]_{M}\right]_{M} & +\left[a_{A}(Z),\left[a_{A}(X), a_{A}(Y)\right]_{M}\right]_{M} \\
& +\left[a_{A}(Y),\left[a_{A}(Z), a_{A}(X)\right]_{M}\right]_{M} .
\end{aligned}
$$

Since $[\cdot, \cdot]_{M}$ satisfies the Jacobi identity, $\rho L=0$ and so $L=0$, because $\rho$ is one-toone. Now, we check the Leibniz rule for $[\cdot, \cdot]_{K}$. We have

$\rho[X+K, f Y+K]_{K}=\left[a_{A}(X), f a_{A}(Y)\right]_{M}=a_{A}(X)(f) a_{A}(Y)+f\left[a_{A}(X), a_{A}(Y)\right]_{M}$.

Then

$$
[X+K, f Y+K]_{K}=a_{A}(X)(f) \rho^{-1}\left(a_{A}(Y)\right)+f \rho^{-1}\left[a_{A}(X), a_{A}(Y)\right]_{M},
$$


which is equivalent to

$$
[X+K, f Y+K]_{K}=\rho(X+K)(f)(Y+K)+f[X+K, Y+K]_{M} .
$$

\section{Linear Hom-Poisson structures}

In this section, we introduce the notion of linear Hom-Poisson structure that is a generalization of the notion of linear Hom-Poisson structure introduced in [6]. Also, we show that there exists a one-to-one correspondence between linear Hom-Poisson structures and Hom-Lie algebroids.

Definition 4.1. Let $\left(A \rightarrow M, \varphi, \phi_{A}\right)$ be a Hom-bundle. A linear Hom-Poisson structure on a Hom-bundle $\left(A^{*} \rightarrow M, \varphi, \phi_{A}^{\dagger}\right)$ is a pair that consists of a bracket of functions

$$
\{\cdot, \cdot\}_{A^{*}}: C^{\infty}\left(A^{*}\right) \times C^{\infty}\left(A^{*}\right) \rightarrow C^{\infty}\left(A^{*}\right)
$$

and the map $\left(\phi_{A}^{\dagger}\right)^{*}: C^{\infty}\left(A^{*}\right) \rightarrow C^{\infty}\left(A^{*}\right)$ (a morphism of the function ring $\left.C^{\infty}\left(A^{*}\right)\right)$, such that the following conditions are satisfied:

(i) $\left(C^{\infty}\left(A^{*}\right),\{\cdot, \cdot\}_{A^{*}},\left(\phi_{A}^{\dagger}\right)^{*}\right)$ is a Hom-Lie algebra,

(ii) $\left\{\psi_{1}, \psi_{2} \psi_{3}\right\}_{A^{*}}=\left(\phi_{A}^{\dagger}\right)^{*}\left(\psi_{2}\right)\left\{\psi_{1}, \psi_{3}\right\}_{A^{*}}+\left\{\psi_{1}, \psi_{2}\right\}_{A^{*}}\left(\phi_{A}^{\dagger}\right)^{*}\left(\psi_{3}\right)$,

(iii) $\{\cdot, \cdot\}_{A^{*}}$ is linear, that is, if $\psi_{1}$ and $\psi_{2}$ are linear functions on $A^{*}$ then $\left\{\psi_{1}, \psi_{2}\right\}_{A^{*}}$ is a linear function.

There exists a one-to-one correspondence between the section space $\Gamma(A)$ and the space of linear functions on $A^{*}$. In fact, if $X \in \Gamma(A)$, then the corresponding linear function $\hat{X}$ is defined by

$$
\hat{X}\left(\omega_{m}\right)=\phi_{A}^{\dagger}(\omega)\left(\phi_{A}(X)\right)\left(\varphi^{-1}(m)\right)
$$

where $\omega_{m} \in A_{m}^{*}$. We have

$$
\begin{aligned}
\hat{X}\left(\omega_{\alpha}(m) e^{\alpha}(m)\right) & =\left(\phi_{A}^{\dagger}\left(\omega_{\alpha} e^{\alpha}\right)\left(\phi_{A}(X)\right)\right)\left(\varphi^{-1}(m)\right) \\
& =\varphi^{*}\left(\omega_{\alpha}\right)\left(\varphi^{-1}(m)\right)\left(\phi_{A}^{\dagger}\left(e^{\alpha}\right)\left(\phi_{A}(X)\right)\right)\left(\varphi^{-1}(m)\right)=\omega_{\alpha}(m) \hat{X}\left(e^{\alpha}(m)\right) .
\end{aligned}
$$

Also

$$
\begin{aligned}
\widehat{f X}\left(\omega_{m}\right) & =\left(\phi_{A}^{\dagger}(\omega)\left(\phi_{A}(f X)\right)\right)\left(\varphi^{-1}(m)\right) \\
& =\left(\phi_{A}^{\dagger}(\omega)\left(\varphi^{*}(f) \phi_{A}(X)\right)\right)\left(\varphi^{-1}(m)\right)=\varphi^{*}(f)\left(\varphi^{-1}(m)\right) \hat{X}\left(\omega_{m}\right) \\
& =f(m) \hat{X}\left(\omega_{m}\right)=f\left(\tau_{A^{*}}(\omega(m))\right) \hat{X}\left(\omega_{m}\right)=\left(f \circ \tau_{A^{*}}\right)\left(\omega_{m}\right) \hat{X}\left(\omega_{m}\right) .
\end{aligned}
$$

Therefore $\widehat{f X}=\left(f \circ \tau_{A^{*}}\right) \hat{X}$. We consider $\left(\phi_{A}^{\dagger}\right)^{*}: C^{\infty}\left(A^{*}\right) \rightarrow C^{\infty}\left(A^{*}\right)$ given by

$$
\left(\left(\phi_{A}^{\dagger}\right)^{*}(F)\right)\left(\omega_{m}\right)=F\left(\left(\phi_{A}^{\dagger}\right)^{-1}(\omega)_{\varphi(m)}\right), \quad \forall F \in C^{\infty}\left(A^{*}\right),
$$

which gives us

$$
\left(\left(\phi_{A}^{\dagger}\right)^{*}(\hat{X})\right)\left(\omega_{m}\right)=\left(\omega\left(\phi_{A}(X)\right)\right)_{m} .
$$

For any $f \in C^{\infty}(M)$, we conclude

$$
\begin{aligned}
\left(\phi_{A}^{\dagger}\right)^{*}(\widehat{f X})\left(\omega_{m}\right) & =\omega\left(\phi_{A}(f X)\right)_{m}=\left(\varphi^{*}(f) \omega\left(\phi_{A}(X)\right)_{m}\right. \\
& =\varphi^{*}(f)(m)\left(\phi_{A}^{\dagger}\right)^{*}(\widehat{X})\left(\omega_{m}\right)=\varphi^{*}(f)\left(\tau_{A^{*}}\left(\omega_{m}\right)\right)\left(\phi_{A}^{\dagger}\right)^{*}(\widehat{X})\left(\omega_{m}\right),
\end{aligned}
$$


i.e.,

$$
\left(\phi_{A}^{\dagger}\right)^{*}(\widehat{f X})=\left(\varphi^{*}(f) \circ \tau_{A *}\right)\left(\phi_{A}^{\dagger}\right)^{*}(\hat{X}) .
$$

Proposition 4.2. Let $\left(\{\cdot, \cdot\}_{A^{*}},\left(\phi_{A}^{\dagger}\right)^{*}\right)$ be a linear Hom-Poisson structure on a Hom-bundle $\left(A^{*} \rightarrow M, \varphi, \phi_{A}^{\dagger}\right)$. If we consider $X \in \Gamma(A)$ and $f, g \in C^{\infty}(M)$, then

(i) $\{\hat{X}, \hat{f}\}_{A^{*}}$ is a basic function with respect to the projection $\tau_{A^{*}}$;

(ii) $\{\hat{f}, \hat{g}\}_{A^{*}}=0$.

Proof. Using 4.4 and property (ii) in Definition 4.1. we get

$$
\left\{\hat{X},\left(f \circ \tau_{A^{*}}\right) \hat{Y}\right\}_{A^{*}}=\left(\varphi^{*}(f) \circ \tau_{A^{*}}\right)\{\hat{X}, \hat{Y}\}_{A^{*}}+\left\{\hat{X},\left(f \circ \tau_{A^{*}}\right)\right\}_{A^{*}}\left(\phi_{A}^{\dagger}\right)^{*}(\hat{Y}) .
$$

Since $\left\{\hat{X},\left(f \circ \tau_{A^{*}}\right) \hat{Y}\right\}_{A^{*}}$ and $\{\hat{X}, \hat{Y}\}_{A^{*}}$ are $\mathbb{R}$-linear functions, we conclude that $\left\{\hat{X},\left(f \circ \tau_{A^{*}}\right)\right\}_{A^{*}}$ is a basic function with respect to $\tau_{A^{*}}$, which implies that (i) holds.

Using (i) and Definition 4.1, we have

$\left\{\left(f \circ \tau_{A^{*}}\right), \hat{Y}\left(g \circ \tau_{A^{*}}\right)\right\}_{A^{*}}=\left(\phi_{A}^{\dagger}\right)^{*}(\hat{Y})\left\{\left(f \circ \tau_{A^{*}}\right),\left(g \circ \tau_{A^{*}}\right)\right\}_{A^{*}}+\left\{\left(f \circ \tau_{A^{*}}\right), \hat{Y}\right\}_{A^{*}} \widehat{\varphi^{*}(g)}$, which is a basic function with respect to $\tau_{A^{*}}$. Therefore we deduce that $\{(f \circ$ $\left.\left.\tau_{A^{*}}\right),\left(g \circ \tau_{A^{*}}\right)\right\}_{A^{*}}=0$. This proves (ii).

Proposition 4.3. If we consider $\phi_{A}\left(\Psi_{\lambda}\right)=\phi_{\lambda}^{\mu} \Psi_{\mu}$, then

$$
\widehat{\Psi_{\alpha}}=\xi_{\alpha}, \quad\left(\phi_{A}^{\dagger}\right)^{*}\left(\xi_{\alpha}\right)=\left(\phi_{\alpha}^{\beta} \circ \tau_{A^{*}}\right) \xi_{\beta}, \quad\left(\left(\phi_{A}^{\dagger}\right)^{*}\right)^{-1} \widehat{\phi_{A}\left(\Psi_{\alpha}\right)}=\widehat{\Psi_{\alpha}} .
$$

Proof. Using 4.1, we obtain

$$
\widehat{\Psi_{\alpha}}\left(\left(\Psi^{\beta}\right)_{m}\right)=\left(\left(\phi_{A}^{\dagger}\right)\left(\Psi^{\beta}\right) \phi_{A}\left(\Psi_{\alpha}\right)\right)_{\varphi^{-1}(m)}=\left(\varphi^{*}\left(\left(\Psi^{\beta}\right)\left(\Psi_{\alpha}\right)\right)\right)_{\varphi^{-1}(m)}=\delta_{\alpha}^{\beta}(m) \text {. }
$$

Thus we get

$$
\widehat{\Psi_{\alpha}}\left(\omega_{m}\right)=\widehat{\Psi_{\alpha}}\left(\omega_{\beta}(m)\left(\Psi^{\beta}\right)_{m}\right)=\omega_{\beta}(m) \delta_{\alpha}^{\beta}=\omega_{\alpha}(m)=\xi_{\alpha}\left(\omega_{m}\right) .
$$

We have

$$
\begin{aligned}
\left(\phi_{A}^{\dagger}\right)^{*}\left(\xi_{\alpha}\right)\left(\omega_{m}\right) & =\xi_{\alpha}\left(\left(\phi_{A}^{\dagger}\right)^{-1}(\omega)_{\varphi(m)}\right)=\xi_{\alpha}\left(\left(\phi_{A}^{\dagger}\right)^{-1}\left(\omega_{\beta} \Psi^{\beta}\right)_{\varphi(m)}\right) \\
& =\xi_{\alpha}\left(\left(\varphi^{*}\right)^{-1}\left(\omega_{\beta}\right)_{\varphi(m)}\left(\phi_{A}^{\dagger}\right)^{-1}\left(\Psi^{\beta}\right)_{\varphi(m)}\right) .
\end{aligned}
$$

On the other hand,

$$
\begin{aligned}
\left(\phi_{A}^{\dagger}\right)^{-1}\left(\Psi^{\beta}\right)\left(\Psi_{\alpha}\right) & =\left(\varphi^{*}\right)^{-1}\left(\Psi^{\beta}\left(\phi_{A}\left(\Psi_{\alpha}\right)\right)\right)=\left(\varphi^{*}\right)^{-1}\left(\Psi^{\beta}\left(\phi_{\alpha}^{\gamma} \Psi_{\gamma}\right)\right) \\
& =\left(\phi_{\alpha}^{\gamma} \circ \varphi^{-1}\right) \delta_{\gamma}^{\beta}=\left(\phi_{\alpha}^{\beta} \circ \varphi^{-1}\right),
\end{aligned}
$$

which gives

$$
\left(\phi_{A}^{\dagger}\right)^{-1}\left(\Psi^{\beta}\right)=\left(\phi_{\alpha}^{\beta} \circ \varphi^{-1}\right) \Psi^{\alpha} .
$$

Setting the above equation in 4.7 implies

$$
\left(\phi_{A}^{\dagger}\right)^{*}\left(\xi_{\alpha}\right)\left(\omega_{m}\right)=\xi_{\alpha}\left(\omega_{\beta}(m) \phi_{\gamma}^{\beta}(m)\left(e^{\gamma}\right)_{\varphi(m)}\right)=\phi_{\alpha}^{\beta}(m) \omega_{\beta}(m)=\phi_{\alpha}^{\beta}(m) \xi_{\beta}\left(\omega_{m}\right) .
$$

The third equation is obtained as follows:

$$
\begin{aligned}
\left.\widehat{\phi_{A}\left(\Psi_{\alpha}\right.}\right)\left(\omega_{m}\right) & =\left(\phi_{A}^{\dagger}(\omega)\left(\phi_{A}^{2}\left(\Psi_{\alpha}\right)\right)\right)_{\varphi^{-1}(m)}=\left(\varphi^{*}\left(\omega\left(\phi_{A}\left(\Psi_{\alpha}\right)\right)\right)\right)_{\varphi^{-1}(m)}=\omega\left(\phi_{A}\left(\Psi_{\alpha}\right)\right)_{m} \\
& =\left(\phi_{A}^{\dagger}\right)^{*}\left(\widehat{\Psi_{\alpha}}\right)\left(\omega_{m}\right) .
\end{aligned}
$$


Corollary 4.4. We have

$$
\left(\phi_{A}^{\dagger}\right)^{*}\left(\widehat{\Psi_{\alpha}}\right)=\left(\phi_{\alpha}^{\beta} \circ \tau_{A^{*}}\right) \widehat{\Psi_{\beta}} .
$$

Theorem 4.5. Let $\left(A, \varphi, \phi_{A},[\cdot, \cdot]_{A}, a_{A}\right)$ be a Hom-Lie algebroid. Then there exists a one-to-one correspondence between the Hom-Lie algebroid and its linear HomPoisson structure. Also, if $\left(\{\cdot, \cdot\}_{A^{*}},\left(\phi_{A}^{\dagger}\right)^{*}\right)$ is a linear Hom-Poisson structure on a Hom-bundle $\left(A^{*} \rightarrow M, \varphi, \phi_{A}^{\dagger}\right)$, then the corresponding Hom-Lie algebroid structure $\left([\cdot, \cdot]_{A}, a_{A}\right)$ on $A$ is characterized by the following conditions:

$$
\begin{aligned}
& {\left[\widehat{X, Y}_{A}=-\{\hat{X}, \hat{Y}\}_{A^{*}},\right.} \\
& a_{A}\left(\phi_{A}(X)\right)(f) \circ \tau_{A^{*}}=\left\{\left(f \circ \tau_{A^{*}}\right), \hat{X}\right\}_{A^{*}}=\varphi^{*}\left(a_{A}(X)\left(\left(\varphi^{*}\right)^{-1}(f)\right)\right) \circ \tau_{A^{*}} .
\end{aligned}
$$

Proof. We consider $\{\cdot, \cdot\}_{A^{*}}$ as a linear Hom-Poisson structure on $A^{*}$. Since $\{\cdot, \cdot\}_{A^{*}}$ is an $\mathbb{R}$-bilinear skew-symmetric bracket, we conclude that $[\cdot, \cdot]_{A}$ is also $\mathbb{R}$-bilinear skew-symmetric. As $\{\cdot, \cdot\}_{A^{*}}$ satisfies the Hom-Leibniz rule, we conclude that $a_{A}(X)$ is a vector field on $M$, for any $X \in \Gamma(A)$. Using (4.8) and the Hom-Leibniz rule we get

$$
\begin{aligned}
a_{A}\left(\phi_{A}(g X)\right)(f) \circ \tau_{A^{*}} & =\left\{\left(f \circ \tau_{A^{*}}\right),\left(g \circ \tau_{A^{*}}\right) \hat{X}\right\}_{A^{*}} \\
& =\left(\phi_{A}^{\dagger}\right)^{*}\left(\left(g \circ \tau_{A^{*}}\right)\right)\left\{\left(f \circ \tau_{A^{*}}\right), \hat{X}\right\}_{A^{*}} \\
& =\left(\phi_{A}^{\dagger}\right)^{*}\left(\left(g \circ \tau_{A^{*}}\right)\right) a_{A}\left(\phi_{A}(X)\right)(f) \circ \tau_{A^{*}},
\end{aligned}
$$

which gives us

$$
a_{A}(g X)(f)=g a_{A}(X)(f),
$$

that is $a_{A}: \Gamma\left(\tau_{A}\right) \rightarrow \Gamma\left(\varphi^{!} \mathrm{T} M\right)$ is a morphism of $C^{\infty}(M)$-modules. Also using 4.5 and 4.8 we obtain

$$
\begin{aligned}
{[\widehat{X, f Y}]_{A} } & =-\{\hat{X}, \widehat{f Y}\}_{A^{*}} \\
& =-\left(\phi_{A}^{\dagger}\right)^{*}\left(\left(f \circ \tau_{A^{*}}\right)\right)\{\hat{X}, \hat{Y}\}_{A^{*}}+a_{A}\left(\phi_{A}(X)\right)(f)\left(\phi_{A}^{\dagger}\right)^{*}(Y) \\
& =\left(\varphi^{*}(f) \circ \tau_{A^{*}}\right)[\widehat{X, Y}]_{A}+a_{A}\left(\phi_{A}(X)\right)(f)\left(\widehat{\phi_{A}(Y)} .\right.
\end{aligned}
$$

Therefore we have

$$
[X, f Y]_{A}=\varphi^{*}(f)[X, Y]_{A}+a_{A}\left(\phi_{A}(X)\right)(f) \phi_{A}(Y) .
$$

Since $\{\cdot, \cdot\}_{A^{*}}$ satisfies the Hom-Jacobi identity, it is easy to see that the Hom-Jacobi identity holds for $[\cdot, \cdot]_{A}$. Therefore $\left([\cdot, \cdot]_{A}, a_{A}\right)$ is a Hom-Lie algebroid structure on $\left(\tau_{A}: A \rightarrow M, \varphi, \phi_{A}\right)$.

Conversely, let $\left([\cdot, \cdot]_{A}, a_{A}\right)$ be a Hom-Lie algebroid structure on a Hom-bundle $\left(\tau_{A}: A \rightarrow M, \varphi, \phi_{A}\right)$. If we consider $x \in M$, then there exists an open subset $U$ of $M$ and a unique linear Poisson structure on the Hom-bundle $\left(\tau_{A^{*}}^{-1}(U) \rightarrow U, \varphi, \phi_{A}^{\dagger}\right)$ such that

$$
\begin{aligned}
& \left.\{\hat{X}, \hat{Y}\}_{\tau_{A^{*}}^{-1}(U)}=-\widehat{[X, Y}\right]_{\tau_{A}^{-1}(U)}, \\
& \left\{\left(f \circ \tau_{A^{*}}\right), \hat{X}\right\}_{\tau_{A^{*}}^{-1}(U)}=a_{\tau_{A}^{-1}(U)}\left(\phi_{A}(X)\right)(f), \\
& \left\{\left(f \circ \tau_{A^{*}}\right),\left(g \circ \tau_{A^{*}}\right)\right\}_{\tau_{A^{*}}^{-1}(U)}=0,
\end{aligned}
$$


for any $X, Y \in \Gamma\left(\tau_{\tau_{A}^{-1}(U)}\right)$ and $f, g \in C^{\infty}(M)$. As $[\cdot, \cdot]_{A}$ satisfies the Hom-Jacobi identity, then the Hom-Jacobi identity holds for $\{\cdot, \cdot\}_{A^{*}}$. So $\left([\cdot, \cdot]_{\tau_{A}^{-1}(U)}, a_{\tau_{A}^{-1}(U)}\right)$ is a Hom-Lie algebroid structure on Hom-bundle $\left(\tau_{\tau_{A}^{-1} U}: \tau_{A}^{-1}(U) \rightarrow M, \varphi, \phi_{\tau_{A}^{-1}(U)}\right)$. Therefore, there exists a unique linear Hom-Poisson structure $\left(\{\cdot, \cdot\}_{A^{*}},\left(\phi_{A}^{\dagger}\right)^{*}\right)$ on $A^{*}$ that satisfies 4.8 .

Let $x^{i}$ denote the local coordinates over a neighborhood $U$ of $M$. If $e_{\alpha}$ is a basis of sections of the Hom-bundle $\left(\tau_{A}^{-1}(U) \rightarrow U, \varphi, \phi_{A}\right)$, then $\left(x^{i}, \xi_{\alpha}\right)$ are the corresponding local coordinates on the Hom-bundle $\left(A^{*} \rightarrow M, \varphi, \phi_{A}^{\dagger}\right)$. By Proposition 4.2 one can easily see that

$$
\left\{\xi_{\alpha}, \xi_{\beta}\right\}_{A^{*}}=-\left(C_{\alpha \beta}^{\gamma} \circ \tau_{A^{*}}\right) \xi_{\gamma}, \quad\left\{x^{i}, \xi_{\alpha}\right\}_{A^{*}}=\left(\phi_{\alpha}^{\beta} a_{\beta}^{i}\right) \circ \tau_{A^{*}}, \quad\left\{x^{i}, x^{j}\right\}_{A^{*}}=0,
$$

where $a_{\beta}^{i}, C_{\alpha \beta}^{\mu}$ are smooth maps on $U$.

Proposition 4.6. Let $\left(\{\cdot, \cdot\}_{A^{*}},\left(\phi_{A}^{\dagger}\right)^{*}\right)$ be a linear Hom-Poisson structure on the Hom-bundle $A^{*}$ and $\left([\cdot, \cdot]_{A}, a_{A}\right)$ be the corresponding Hom-Lie algebroid structure on the Hom-bundle $\left(\tau_{A}: A \rightarrow M, \varphi, \phi_{A}\right)$. If $x^{i}$ are local coordinates on an open subset $U$ of $M$ and $e_{\alpha}$ is a basis of sections of the Hom-bundle $\tau_{A}^{-1}(U) \rightarrow U$, then

$$
\left[\Psi_{\alpha}, \Psi_{\beta}\right]_{A}=C_{\alpha \beta}^{\lambda} \Psi_{\lambda}, \quad a_{A}\left(\phi_{A}\left(\Psi_{\alpha}\right)\right)=\phi_{\alpha}^{\beta} a_{\beta}^{i} \frac{\partial}{\partial x^{i}},
$$

where $C_{\alpha \beta}^{\lambda}, \phi_{\alpha}^{\beta} a_{\beta}^{i}$ are called the local structure functions of the Hom-Lie algebroid structure $\left([\cdot, \cdot]_{A}, a_{A}\right)$ on the Hom-bundle A.

Proof. We have

$$
\left[\widehat{\Psi_{\alpha}, \Psi_{\beta}}\right]_{A}=-\left\{\widehat{\Psi_{\alpha}}, \widehat{\Psi_{\beta}}\right\}_{A^{*}}=\left(C_{\alpha \beta}^{\gamma} \circ \tau_{A^{*}}\right) \widehat{\Psi_{\gamma}} .
$$

Contracting the above equation by $\Psi^{\nu}$ and using 4.6 we obtain

$$
\Psi^{\nu}\left(\left[\Psi_{\alpha}, \Psi_{\beta}\right]_{A}\right)=C_{\alpha \beta}^{\nu}
$$

Also we get

$$
\left.a_{A}\left(\phi_{A}\left(\Psi_{\alpha}\right)\right)(f) \circ \tau_{A^{*}}=a_{A}\left(\phi_{A}\left(\Psi_{\alpha}\right)\right)(f)\right) \circ \tau_{A^{*}}=\left\{f \circ \tau_{A^{*}}, \widehat{\Psi_{\alpha}}\right\}_{A^{*}}=\left(\phi_{\alpha}^{\beta} a_{\beta}^{i}\right) \circ \tau_{A^{*}}
$$

Now we shall compute the Hamiltonian vector fields $X_{x^{i}}, X_{\xi_{\alpha}}$ on $A^{*}$. Using the definition we get

$$
X_{x^{i}}\left(x^{j}\right)=\left\{x^{i}, x^{j}\right\}=0, \quad X_{x^{i}}\left(\xi_{\alpha}\right)=\left\{x^{i}, \xi_{\alpha}\right\}=\left(a_{\beta}^{i} \phi_{\alpha}^{\beta}\right) \circ \tau_{A^{*}} .
$$

So, one can get

For $X_{\xi_{\alpha}}$ we have

$$
X_{x^{i}}=\left(a_{\beta}^{i} \phi_{\alpha}^{\beta}\right) \circ \tau_{A^{*}} \frac{\partial}{\partial \xi_{\alpha}} .
$$

$$
X_{p_{\alpha}}\left(x^{i}\right)=\left\{\xi_{\alpha}, x^{i}\right\}=-\left(a_{\beta}^{i} \phi_{\alpha}^{\beta}\right) \circ \tau_{A^{*}}, \quad X_{p_{\alpha}}\left(\xi_{\beta}\right)=\left\{\xi_{\alpha}, \xi_{\beta}\right\}=-\left(C_{\alpha \beta}^{\gamma} \circ \tau_{A^{*}}\right) \xi_{\gamma} .
$$


So

$$
X_{\xi_{\alpha}}=-\left(a_{\beta}^{i} \phi_{\alpha}^{\beta}\right) \circ \tau_{A^{*}} \frac{\partial}{\partial x^{i}}-\left(C_{\alpha \beta}^{\gamma} \circ \tau_{A^{*}}\right) \xi_{\gamma} \frac{\partial}{\partial \xi_{\beta}} .
$$

Let $v=v^{\alpha} \Psi_{\alpha} \in \Gamma(A)$ be a local section and define $f_{v}: A^{*} \rightarrow \mathbb{R}$ in the usual way

$$
f_{v}(x, \xi)=v^{\alpha}(x) \xi_{\alpha}(x, \xi) \quad \text { or } \quad f_{v}=\left(v^{\alpha} \circ \tau_{A^{*}}\right) \xi_{\alpha},
$$

where $\xi=\sum_{\alpha} \xi_{\alpha} \Psi^{* \alpha}$. Now, we compute $X_{f_{v}}$ as follows:

$$
X_{f_{v}}\left(x^{i}\right)=-X_{x^{i}}\left(f_{v}\right)=-\left(a_{\beta}^{i} \phi_{\alpha}^{\beta} \varphi^{*}\left(v^{\alpha}\right)\right) \circ \tau_{A^{*}}
$$

and

$$
\begin{aligned}
X_{f_{v}}\left(\xi_{\alpha}\right) & =\left\{f_{v}, p_{\alpha}\right\}=\left\{\left(v^{\lambda} \circ \tau_{A^{*}}\right) \xi_{\lambda}, \xi_{\alpha}\right\} \\
& =\left(\phi_{\lambda}^{\gamma} \circ \tau_{A^{*}}\right) \xi_{\gamma}\left\{v^{\lambda} \circ \tau_{A^{*}}, \xi_{\alpha}\right\}+\left(\varphi^{*}\left(v^{\lambda}\right) \circ \tau_{A^{*}}\right)\left\{\xi_{\lambda}, \xi_{\alpha}\right\} \\
& =\left(\left(\phi_{\lambda}^{\gamma} a_{\beta}^{i} \phi_{\alpha}^{\beta} \frac{\partial v^{\lambda}}{\partial x^{i}}\right) \circ \tau_{A^{*}}\right) \xi_{\gamma}-\left(\left(\varphi^{*}\left(v^{\lambda}\right) C_{\lambda \alpha}^{\gamma}\right) \circ \tau_{A^{*}}\right) \xi_{\gamma} .
\end{aligned}
$$

So we have

$$
\begin{aligned}
X_{f_{v}}= & -\left(a_{\beta}^{i} \phi_{\alpha}^{\beta} \varphi^{*}\left(v^{\alpha}\right)\right) \circ \tau_{A^{*}} \frac{\partial}{\partial x^{i}} \\
& +\left(\left(\phi_{\lambda}^{\gamma} a_{\beta}^{i} \phi_{\alpha}^{\beta} \frac{\partial v^{\lambda}}{\partial x^{i}}\right) \circ \tau_{A^{*}} \xi_{\gamma}-\left(\varphi^{*}\left(v^{\lambda}\right) C_{\lambda \alpha}^{\gamma}\right) \circ \tau_{A^{*}} \xi_{\gamma}\right) \frac{\partial}{\partial \xi_{\alpha}} .
\end{aligned}
$$

Since $\left(\tau_{A^{*}}\right)_{*}\left(\frac{\partial}{\partial x^{i}}\right)=\frac{\partial}{\partial x^{i}}$ and $\left(\tau_{A^{*}}\right)_{*}\left(\frac{\partial}{\partial \xi_{\alpha}}\right)=0$, from the above equation we get

$$
\left(\tau_{A^{*}}\right)_{*}\left(X_{f_{v}}\right)=-\left(a_{\beta}^{i} \phi_{\alpha}^{\beta} \varphi^{*}\left(v^{\alpha}\right)\right) \circ \tau_{A^{*}} \frac{\partial}{\partial x^{i}}=-a_{A}\left(\phi_{A}(v)\right) .
$$

Let $v=v^{\alpha} \Psi_{\alpha}$ and $w=w^{\beta} \Psi_{\beta}$. Then one can compute $\left\{f_{v}, f_{w}\right\}$ as follows:

$$
\begin{aligned}
\left\{f_{v}, f_{w}\right\}= & \left\{v^{\alpha} \xi_{\alpha}, w^{\beta} \xi_{\beta}\right\} \\
= & \left(\phi_{\alpha}^{\lambda} \circ \tau_{A^{*}}\right) \xi_{\lambda}\left\{v^{\alpha}, w^{\beta} \xi_{\beta}\right\}+\varphi^{*}\left(v^{\alpha}\right) \circ \tau_{A^{*}}\left\{\xi_{\alpha}, w^{\beta} \xi_{\beta}\right\} \\
= & \left(\left(\phi_{\alpha}^{\lambda} \varphi^{*}\left(w^{\beta}\right)\right) \circ \tau_{A^{*}}\right) \xi_{\lambda}\left\{v^{\alpha}, \xi_{\beta}\right\}+\left(\left(\phi_{\alpha}^{\lambda} \phi_{\beta}^{\gamma}\right) \circ \tau_{A^{*}}\right) \xi_{\lambda} \xi_{\gamma}\left\{v^{\alpha}, w^{\beta}\right\} \\
& +\left(\varphi^{*}\left(v^{\alpha}\right) \varphi^{*}\left(w^{\beta}\right)\right) \circ \tau_{A^{*}}\left\{\xi_{\alpha}, \xi_{\beta}\right\}+\left(\left(\varphi^{*}\left(v^{\alpha}\right) \phi_{\beta}^{\gamma}\right) \circ \tau_{A^{*}}\right) \xi_{\gamma}\left\{\psi_{\alpha}, w^{\beta}\right\} \\
= & \left(\left(\phi_{\alpha}^{\lambda} a_{\mu}^{i} \phi_{\beta}^{\mu} \varphi^{*}\left(w^{\beta}\right) \frac{\partial v^{\alpha}}{\partial x^{i}}\right) \circ \tau_{A^{*}}\right) \xi_{\lambda}-\left(\left(\varphi^{*}\left(v^{\alpha}\right) \varphi^{*}\left(w^{\beta}\right) C_{\alpha \beta}^{\gamma}\right) \circ \tau_{A^{*}}\right) \xi_{\gamma} \\
& -\left(\left(\varphi^{*}\left(v^{\alpha}\right) \phi_{\beta}^{\gamma} a_{\mu}^{i} \phi_{\alpha}^{\mu} \frac{\partial w^{\beta}}{\partial x^{i}}\right) \circ \tau_{A^{*}}\right) \xi_{\gamma} .
\end{aligned}
$$

It is a straightforward computation to see that

$$
\begin{aligned}
{[v, w]=- } & \left(\left(\phi_{\alpha}^{\lambda} a_{s}^{i} \phi_{\beta}^{s} \varphi^{*}\left(w^{\beta}\right) \frac{\partial v^{\alpha}}{\partial x^{i}}-\varphi^{*}\left(v^{\alpha}\right) \varphi^{*}\left(w^{\beta}\right) C_{\alpha \beta}^{\lambda}\right.\right. \\
& \left.\left.-\varphi^{*}\left(v^{\alpha}\right) \phi_{\beta}^{\lambda} a_{u}^{i} \phi_{\alpha}^{u} \frac{\partial w^{\beta}}{\partial x^{i}}\right) \circ \tau_{A^{*}}\right) \Psi_{\lambda},
\end{aligned}
$$


and so

$$
\begin{gathered}
f_{[v, w]}=-\left(\left(\phi_{\alpha}^{\lambda} a_{s}^{i} \phi_{\beta}^{s} \varphi^{*}\left(w^{\beta}\right) \frac{\partial v^{\alpha}}{\partial x^{i}}-\varphi^{*}\left(v^{\alpha}\right) \varphi^{*}\left(w^{\beta}\right) C_{\alpha \beta}^{\lambda}\right.\right. \\
\left.\left.-\varphi^{*}\left(v^{\alpha}\right) \phi_{\beta}^{\lambda} a_{u}^{i} \phi_{\alpha}^{u} \frac{\partial w^{\beta}}{\partial x^{i}}\right) \circ \tau_{A^{*}}\right) \xi_{\lambda} .
\end{gathered}
$$

(4.9) and 4.10 imply that $-f_{[v, w]}=\left\{f_{v}, f_{w}\right\}=\left\{-f_{v},-f_{w}\right\}$. Using the above calculations we have the following theorem.

Theorem 4.7. The assignment $v \longmapsto-f_{v}$ defines a Lie algebra homomorphism $\left(\Gamma(A),[\cdot, \cdot]_{A}\right) \rightarrow\left(C^{\infty}\left(A^{*}\right),\{\cdot, \cdot\}_{a}\right)$. Moreover, if $X_{f_{v}}$ denotes the Hamiltonian vec-

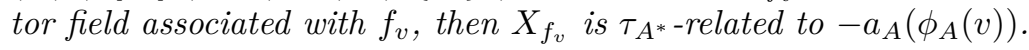

\section{REFERENCES}

[1] L. Cai, J. Liu and Y. Sheng, Hom-Lie algebroids, Hom-Lie bialgebroids and Hom-Courant algebroids, J. Geom. Phys. 121 (2017), 15-32. MR 3705378

[2] J.-P. Dufour and N. T. Zung, Poisson structures and their normal forms, Progress in Mathematics, vol. 242, Birkhäuser Verlag, Basel, 2005. MR 2178041

[3] R.L. Fernandes, Lie algebroids, holonomy and characteristic classes, Adv. Math. 170 (2002), no. 1, 119-179. MR 1929305.

[4] C. Laurent-Gengoux and J. Teles, Hom-Lie algebroids, J. Geom. Phys. 68 (2013), 69-75. MR 3035115

[5] J. Liu, Y. Sheng, C. Bai and Z. Chen, Left-symmetric algebroids, Math. Nachr. 289 (2016), no. 14-15, 1893-1908. MR 3563909

[6] M. de León, J. C. Marrero, D. Martín de Diego, Linear almost Poisson structures and Hamilton-Jacobi equation. Applications to nonholonomic mechanics, J. Geom. Mech. 2 (2010), no. 2, 159-198. MR 2660714

[7] K. C. H. Mackenzie, General theory of Lie groupoids and Lie algebroids, London Math. Soc. Lecture notes series, vol. 213, Cambridge University Press, Cambridge, 2005. MR 2157566

[8] S. Merati, M. R. Farhangdoost, Representation up to homotopy of Hom-Lie algebroids, Int. J. Geom. Methods Mod. Phys. 15 (2018), no. 5, 1850074, 13 pp. MR 3786506.

[9] E. Peyghan and L. Nourmohammadifar, Para-Kähler hom-Lie algebras, J. Algebra Appl. 18 (2019), no. 3, 1950044, 24 pp. MR 3924822

[10] E. Peyghan and L. Nourmohammadifar, Hom-left symmetric algebroids, Asian-Eur. J. Math. 11 (2018), no. 2, 1850027, 24 pp. MR 3786367

[11] Y. Sheng and C. Bai, A new approach to hom-Lie bialgebras, J. Algebra 399 (2014), 232-250. MR 3144586

[12] S. Vacaru, Clifford-Finsler algebroids and nonholonomic Einstein-Dirac structures, J. Math. Phys. 47 (2006), no. 9, 093504, 20 pp. MR 2263658

[13] S. Vacaru, Finsler and Lagrange geometries in Einstein and string gravity, Int. J. Geom. Methods Mod. Phys. 5 (2008), no. 4, 473-511. MR 2428807.

[14] L. Bubuianu and S. I. Vacaru, Dynamical equations and Lagrange-Ricci flow evolution on prolongation Lie algebroids, Canad. J. Phys. 97 (2019), no. 2, 145-154. 
[15] S. Vacaru, Nonholonomic algebroids, Finsler geometry, and Lagrange-Hamilton spaces, Math. Sci. (Springer) 6 (2012), Art. 18, 33 pp. MR 3064449

[16] S. Vacaru, Almost Kähler Ricci flows and Einstein and Lagrange-Finsler structures on Lie algebroids, Mediterr. J. Math. 12 (2015), no. 4, 1397-1427. MR 3416867

E. Peyghan ${ }^{凶}$

Department of Mathematics, Faculty of Science, Arak University, Arak 38156-8-8349, Iran

e-peyghan@araku.ac.ir

A. Baghban

Department of Mathematics, Faculty of Science, Azarbaijan Shahid Madani University, Tabriz 5375171379 , Iran

amirbaghban87@gmail.com

E. Sharahi

Department of Mathematics, Faculty of Science, Arak University, Arak 38156-8-8349, Iran

e-sharahi@phd.araku.ac.ir

Received: May 20, 2018

Accepted: November 30, 2018 